\title{
Пандемия и факторы роста производительности труда
}

\author{
В. В. Гридасова ${ }^{1}$, В. В. Материева ${ }^{2 凶}$
}

${ }_{1,2}^{2}$ Воронежский государственный университет, Университетская пл., 1, 394018, Воронеж, Российская Федерация

Для цитирования: Матершева В. В., Гридасова В. В. Пандемия и факторы роста производительности труда // Вестник Воронежского государственного университета. Серия: Экономика и управление. 2021. № 1. C. 13-24. DOI: 10.17308/econ.2021.1/3346

Предмет. Изучение проблем производительности труда всегда было важной темой исследований в различных странах. Особенностью данной статьи является то, что она включает обзор работ преимущественно зарубежных авторов, посвященных исследованию факторов, влияющих на производительность труда и их проявление в условиях пандемии.

Цели. Выделены две основные цели работы: изучить определения некоторых авторов относительно категории «продуктивность» в их исследованиях; найти набор тех факторов, наличие или отсутствие которых может улучшить состояние продуктивности любой организации.

Методология. В процессе достижения поставленной цели использовались методы научного познания: диалектический; анализа и синтеза, восхождения от абстрактного к конкретному, моделирование. С помощью систематического обзора будет восполнен пробел, созданный традиционными повествовательными обзорами, из-за использования авторами субъективной и предвзятой методологии.

Результаты. Выполнен систематический анализ статей, доступных в научных журналах, и определены соответствующие факторы, влияющие на производительность труда. В ходе данной работы выполнена основная цель - объединены различные факторы, указанные в нескольких исследованиях, для лучшего понимания определения производительности и дальнейшего продвижения будущих исследований в области повышения производительности. Классифицированы факторы в семи измерениях и предложена модель повышения производительности труда. Показано, как один идентифицированный фактор взаимодействует или влияет на другой выявленный фактор.

Выводы. Системный обзор многочисленных и разнообразных исследований, осуществляемых в последние десятилетия зарубежными учеными, проведенный в рамках данной статьи, позволил определить влияние различных факторов друг на друга, их взаимодействие и иерархию. Своевременный учет возможностей проявления тех или иных факторов, действующих на различных уровнях, будет способствовать эффективному управлению как на макро-, так и микро-уровне, росту производительности труда.

Ключевые слова: классификация факторов производительности труда, модель повышения производительности труда.

\section{Введение}

В условиях последствий пандемии и обострения глобальной конкуренции особенно остро встает вопрос важности производительности труда и ее измерения. Производительность - основной фактор экономического роста, один из наиболее важных аспектов, который может улучшить или разрушить любую организацию.

Обзор литературы сделан на основе анализа большого количества зарубежных работ, посвященных данной проблеме. Они включают в себя разные определения производительности и отличаются по глубине и объему иссле- дований. В результате можно сделать вывод, что не существует единого метода, использование которого позволило бы достигнуть высокой производительности. Исследование Millea and Fuess [30] показывает, что отдельные факторы могут быть движущими силами производительности в одной стране, в то время как в другой они таковыми не станут. Таким образом, несмотря на значительное количество исследований, данная проблема остается актуальной в постоянно и быстро меняющемся мире и существующие исследования не в полной мере раскрывают воздействие факторов на производительность.

(C) Матершева В. В., Гридасова В. В., 2021

Вестник ВГУ. Серия: Экономика и управление. 2021. № 1. С. 13-24. 
Данная работа направлена на восполнение этого пробела путем систематического анализа статей, доступных в научных журналах, и определения соответствующих факторов, которые влияют на производительность. Основной целью настоящей статьи является систематизация и объединение различных факторов из множества исследований в одно, чтобы результат был полезен для будущих исследований в области повышения производительности и учета степени их воздействия при различных обстоятельствах, например, в условиях поразившей в настоящее время мировую экономику ковидной пандемии.

Данное исследование состоит из систематического анализа зарубежной литературы, посвященной продуктивности труда, для того чтобы определить набор факторов, влияющих на производительность. Под факторами влияния мы понимаем элементы, в отношении которых можно предпринять действия для улучшения производительности фирмы. Основные цели исследования:

- изучить определения некоторых авторов относительно категории «продуктивность» в их исследованиях;

- найти набор тех факторов, наличие или отсутствие которых может улучшить состояние продуктивности любой организации.

\section{Материалы и методы исследования}

В процессе достижения поставленных целей использовались методы научного познания: диалектический; анализа и синтеза, восхождения от абстрактного к конкретному, моделирование. Исследование построено на изучении многолетних и многочисленных исследований, проводимых зарубежными учеными, в том числе на основе не переведенных ранее на русский язык источников.

\section{Обсуждение результатов}

Чтобы лучше понять проблему, необходимо сделать несколько уточнений. Нет общепринятого определения производительности труда, так как разные страны и многие авторы предлагали свои критерии для определения эффективности труда. Каждая отрасль использует собственные модификации, спецификации или уровень детализации, ориентированные на особые потребности в разработке новых показателей производительности труда (см. напр. Симонова [1], Фомина [2], Bureš and Stropková [10]). Mohanty [31] дал 12 различных определений производительности с классификацией определений на макро- и микроуровень. Bernolak [8] и Hannula [20] подробно обсуждают использование и применимость различных определений. В современной литературе представлены различные подходы к трактовке производительности труда (табл. 1).

В рамках настоящего исследования производительность в широком смысле определяется как эффективное использование ресурсов для достижения поставленных целей. Следует выделить несколько аспектов в отношении приведенного выше определения:

1) эффективное использование неэффективных ресурсов;

2) цели должны быть поставлены, определены и должны быть ясны;

3) ресурсы должны существовать и помогать в достижении цели.

Следует отметить, что данный обзор ориентирован только на поиск факторов, которые находятся под контролем организации (определены как внутренние факторы). Несмотря на то что внешние факторы обычно рассматриваются как выходящие за рамки компетенции организации, они могут находиться под кон-

Т а бл и ц а 1

Современные трактовки производительности (продуктивности)

\begin{tabular}{|l|c|}
\hline \multicolumn{1}{|c|}{ Производительность - это ... } & Источник \\
\hline $\begin{array}{l}\text { Отношение добавленной стоимости к человеко-часам или общей сум- } \\
\text { ме стоимость продаж }\end{array}$ & Hannula [20] \\
\hline Добавленная стоимость на одного работника & Enshassi et al. [16] \\
\hline Отношение выход-вход & Durdyev el al. [15] \\
\hline $\begin{array}{l}\text { Количество работы, произведенной за человеко-час, час оборудования } \\
\text { или отработанный час экипажа }\end{array}$ & Nasirzadeh and Nojedehi [32] \\
\hline $\begin{array}{l}\text { Сотношение между выполненной работой и затраченным временем } \\
\text { на выполнение проекта }\end{array}$ & Abad et al. [1] \\
\hline Продажи, разделенные на сотрудников & Calcagnini et al. [11] \\
\hline Реальный объем производства в час работы & Bureš and Stropková [10] \\
\hline Количество товаров, произведенных в пределах единицы рабочей силы
\end{tabular}


тролем промышленных регулирующих органов и лиц, определяющих политику в правительстве, которые могут стать потенциальной аудиторией этой статьи.

Рассмотрим содержание выделенные факторы производительности труда и их содержание.

1. Условия труда. Рабочее место должно удовлетворять стандартам охраны здоровья и обеспечения безопасности труда, а также соответствовать основным санитарным правилам и нормам. Abad et al. [1] обнаружил повышение производительности труда после внедрения в организациях Системы менеджмента охраны здоровья и обеспечения безопасности труда (OHSAS 18001).

2. Заработная плата традиционно является важным фактором производительности труда сотрудников. Об определении величины справедливой заработной платы ведутся длительные дискуссии в Wygant [39]. Должна ли она быть такой же, как в среднем по рынку, должна ли она состоять из высокой стимулирующей части и высокой переменной оплаты труда? Кроме того, были дискуссии о том, влияет ли заработная плата на производительность или реагирует на нее (см., напр.: Feldstein [17], Millea and Fuess [30]). Zhang and Liu [40] анализируют эволюционирующую модель соотношения и распределения заработной платы и производительности труда в обрабатывающем секторе промышленности Китая. Приведенный в исследовании количественный анализ показал, что структура собственности капитала, соотношение капитала и рабочей силы, размер фирмы оказывают значимое влияние на уровень заработной платы.

3. Корпоративная среда. Выстраивание эффективной рабочей среды позволяет сконцентрировать внимание на ключевых задачах организации. Цели организации должны корректно интерпретироваться сотрудниками, а сбалансированное распределение полномочий обеспечить эффективность командной работы по их достижению. Организация, принятие решений в которой строится лишь на консенсусах, не способна понять динамичные изменения в бизнес-среде и не может развиваться. Свобода принятия решений, ответственность, подотчетность и полномочия должны быть предоставлены сотрудникам для того, чтобы инновационная среда способствовала развитию культуры предпринимательства. Mohanty [31] отмечает, что работники должны быть свободны в самовыражении и не должны бояться конфликтов.

4. Организационная структура и культура: устаревшая организационная структура явля- ется еще одной причиной низкой производительности труда. Усилия менеджеров должны быть направлены на создание сплоченной и компактной организационной структуры для поддержки производительности, а также препятствования развитию бюрократических процедур. Mathew [28] подчеркивает связь организационной культуры с качеством и производительностью, уделяя особое внимание фирмам, производящим программное обеспечение в Индии. С одной стороны, организационная культура должна быть направлена на поддержку сотрудников из разных стран. Однако Roberge and van Dick [35] отмечают негативное влияние культурного разнообразия на эффективность работы в команде. Видение организации, миссия и утверждение ценностей должны быть связаны с системой ценностей и личными целями сотрудников.

5. Обучение, повышение квалификации и развитие сотрудников. Bower and Hilgard [9] отмечают, что обучение необходимо тогда, когда для определенного стимула, связанного с работой, сотрудники реагируют по-разному на качественное улучшение от своих реакций на подобные стимулы в прошлом. Организация должна обучать и мотивировать своих сотрудников таким образом, чтобы способствовать достижению организационных целей, а также помогать в индивидуальном карьерном и личностном развитии сотрудника. С развитием технологий сотрудники могут с легкостью продолжить обучение на протяжении всей жизни вне зависимости от своего местонахождения. MacDuffie and Kochan [26] обнаружили, что фирмы с высоким уровнем инвестиций в обучение сотрудников показали более высокий уровень производительности по сравнению с фирмами с низким уровнем. Schonewille [36] отмечает положительное влияние обучения на уровень производительности труда.

6. Кадровая политика организации. Различными авторами было проведено несколько глубоких исследований о влиянии кадровой политики и систем оплаты труда на производительность. Arthur [4] исследовал сталелитейные заводы, имеющие гибкие системы контроля и управления персоналом, и обнаружил что они имеют более высокую производительность, чем на сталелитейных заводах, имеющих более жесткие методы управления персоналом. Согласно Huselid [22], практика высокопроизводительной работы в области подбора персонала, оценки результатов деятельности, поощрительных выплат, разработки должностных инструк- 
ций, прозрачности процесса рассмотрения жалоб, обмена информацией, оценки отношения к работе, участия в управлении трудовыми ресурсами, обучения и развития, разработка критериев продвижения по службе представляют собой широкую область, которая должна быть охвачена HR-политикой. Gruman и Saks [18] полагают, что повышение эффективности работы может быть наилучшим образом достигнуто путем ориентирования системы управления эффективностью работы на поощрение вовлеченности сотрудников. Koch and McGrath [23] по результатам исследования 319 бизнес-единиц установили, что HR-политика имеет значение для повышения производительности труда. Согласно Beauregard and Henry [6] баланс между работой и личной жизнью может привести к экономии затрат для организации, так как сотрудники могут работать в течение более продолжительного времени в связи с сокращением времени на дорогу. Особенно актуальным стал данный вопрос в связи с переводом значительного количества фирм на удаленную работу, где граница между личным и рабочим временем оказалась достаточно размытой. В результате рабочее время достигало 15 часов в день, в том числе в выходные. Künn-Nelen et al. [24] утверждают, что фирмы с высокой долей сотрудников, работающих неполный рабочий день, более продуктивны по сравнению с фирмами с низкой долей таких сотрудников.

7. Уровень внедрения технологий в организации по отношению к отрасли. Производительность также зависит от уровня внедрения новейших технологий, присутствующих на данном отраслевом рынке. Kwong et al. [25] описывают серию отраслевых проектов, включающих моделирование, планирование и мониторинг средств гибкой производственной системы (FMS) в распределенной среде на основе интернет-технологий. Hasan [21] изучает влияние импортных и отечественных технологий на производительность индийских промышленных предприятий.

8. Четкость целеполагания и планирования в организации. Бизнес-цели должны быть понятны всем сотрудникам, и необходимо работать всем вместе, чтобы их достигнуть. Mohanty [31] утверждает, что любое изменение бизнес-цели или задачи должно последовательно сообщаться сверху вниз так, чтобы необходимые шаги были предприняты для достижения желаемого результата.

9. Трудоголизм и профессиональное выгорание. Вопросы трудоголизма и выгорания исследованы в работе Dall'Ora et al. [13]. По данным Стэнфордской высшей школы бизнеса в США выгорание на рабочем месте требует дополнительных расходов на здравоохранение от 125 до 190 миллиардов долларов в год. В Великобритании выгорание сотрудников или связанный с работой стресс и психические заболевания составляют более половины случаев отсутствия на работе и обходятся британским предприятиям примерно в 26 миллиардов фунтов стерлингов в год. В качестве методов, действенных против выгорания сотрудников, но позволяющих сохранить уровень производительности, Mefford [29] выделяет инвестиции в свое офисное пространство, возможность более короткого рабочего дня, установление льгот для сотрудников, справедливую заработную плату, тимбилдинг.

10. Ментальная и физическая стабильность работников. Последствия старения рабочей силы для производительности труда европейских работодателей были изучены компанией Conen [12]. Работник должен быть физически и психически стабилен, чтобы справляться с работой, только тогда он сможет полностью реализовать свой потенциал.

11. Мотивация, энтузиазм и прокрастинация. Работник должен чувствовать достаточную мотивацию к пониманию целей и задач организации и проявлять энтузиазм к принятию на себя ответственности за любую новую сложную работу, с которой он столкнется. Масеу и Schneider [27] отмечают, что существует множество определений этой концепции, но все они содержат информацию, что вовлеченность сотрудников желательна. Она имеет организационную цель и включает как психологические, так и поведенческие аспекты. Zwick [41] показал, что положительные эффекты в динамике производительности от вовлечения работников цеха сильнее в учреждениях с рабочими союзами.

12. Уровень образования сотрудников. Образование формирует мышление. Работник должен быть достаточно образован, чтобы понимать нюансы работы, которая ему поручена, и должен уметь применять этот навык для улучшения качества или снижения себестоимости продукции [36].

13. Отношение, навыки и компетенции работников. Позитивное отношение порождает способность рисковать. Основные ценности сотрудника формирует реакцию на различные стимулы. Действия формируют привычку, привычка делает характер, характер отражает ценности. 
14. Уровень конкуренции в отрасли. Производительность может также повыситься за счет конкуренции в отрасли, сопровождающейся развитием и распространением эффективных технологий. Wagner [38] утверждает, что организации экспортного бизнеса, сталкивающиеся с внешней конкуренцией, могут быть более продуктивными, чем другие, по двум причинам: обслуживание более крупного рынка может позволить фирме воспользоваться любой экономией от масштаба производства или обеспечить некоторое сокращение внутренних колебаний спроса; фирмы, активно работающие на внешних рынках, подвержены более жесткой конкуренции и должны совершенствоваться быстрее, чем те, которые продают свою продукцию только внутри страны.

15. Наличие регулирующего органа в отрасли. Doloi et al. [14], Gunasekaran et al. [19] отмечают, что деятельность регулирующих органов отраслевого уровня может повысить производительность за счет повышения осведомленности о передовом опыте, стандартах качества, программах технического обслуживания, статистике производительности, идеальных производственных процессах и системах.

16. Макроэкономика страны. Производительность организации также зависит от макроэкономической среды фирмы [31]. Себестоимость продукции довольно сильно зависит от уровня инфляции и безработицы, которая в дальнейшем зависит от ВВП и ВНП страны. Демографические показатели также играют ключевую роль в доступности ключевых ресурсов.

17. Государственное регулирование в отрасли. Реформы, проводимые правительством либо путем проведения новой политики, либо путем повышения уровня приобретения и передачи технологий, играют ключевую роль в повышении производительности труда. Pandey и Dong [34] пришли к выводу, что на институциональные изменения в Китае может приходиться значительная часть прироста производительности обрабатывающих отраслей. В работе Nataraj [33] изучалось влияние либерализации тарифов на производительность предприятий. Исследование показывает, что одностороннее снижение в Индии тарифов на готовую продукцию привело к повышению средней производительности небольших фирм.

18. Миграция квалифицированной рабочей силы между странами. Wacker et al. [37] указывает на то, что миграция рабочей силы может привести к «утечке мозгов» из одной страны и в то же время сделать другую страну богатой трудовыми ресурсами. Это может сказаться на стоимости рабочей силы и, следовательно, на производительности труда.

19. Эволюция передовых практик и технологических разработок. Hasan [21] продемонстрировал как обмен лучшими практиками и технологиями мирового класса может привести к заметному повышению уровня производительности как на организационном, так и на отраслевом уровнях.

20. Макроэкономика мира. Любое динамическое изменение в макроэкономике мира может привести к изменению производительности фирмы. Такие международные события, как рецессия, изменение цен на нефть, террористические акты и войны, стихийные бедствия могут кардинально изменить рыночный спрос и повлиять на производительность фирмы (см., напр.: Mohanty [31]).

В табл. 2 представлено распределение приведенных факторов по пяти категориям.

На рисунке представлена модель, отображающая воздействие факторов на производительность труда.

Влияние «зонтика факторов» можно проиллюстрировать, рассмотрев воздействие происходящих в настоящее время в мировой экономике процессов на все уровни принятия решений. Так, эпидемия, вызванная вирусом Covid-19 (международный фактор) оказывает влияние на макроэкономику страны и уровень государственного регулирования, вызвав необходимость осуществления мер государственной поддержки предприятий наиболее пострадавших отраслей. Так, в Германии предприятия малого бизнеса весной 2020 г. получили единовременные выплаты в размере 9 тыс. евро, в РФ были выданы субсидии для работников малых предприятий в размере минимальной оплаты труда (12 130 руб.) и т. д.

Пандемия вызвала массовый переход на «удаленку». В результате качество и способы организации дистанционной работы оказали существенное влияние на производительность труда работников, на эффективность принятия управленческих решений.

Исследования Всемирного банка свидетельствуют, что пандемия необратимо повлияла на рынки труда всех стран. Экономики с доходом выше среднего предсказывают переход на удаленку более 25 \% работников. В ИТ-отрасли переход на удаленку ожидает 74 \% работников, причем развитые страны будут снижать расходы на рабочую силу, привлекая зарубежных сотрудников. 
Категоризация факторов производительности труда

\begin{tabular}{|c|c|}
\hline Категория & Факторы \\
\hline \multirow{5}{*}{$\begin{array}{l}\text { 1. Внутренние по } \\
\text { отношению к сотруднику }\end{array}$} & Ментальная и физическая стабильность работников \\
\hline & Мотивация, энтузиазм и прокрастинация \\
\hline & Уровень образования сотрудника \\
\hline & Отношение, навыки и компетенции сотрудника \\
\hline & Трудоголизм и профессиональное выгорание \\
\hline \multirow{8}{*}{$\begin{array}{l}\text { 2. Внутренние по отношению } \\
\text { к организации, но внешние } \\
\text { по отношению к сотруднику }\end{array}$} & Условия труда \\
\hline & Заработная плата \\
\hline & Корпоративная среда \\
\hline & Организационная структура и культура \\
\hline & Обучение, повышение квалификации и развитие сотрудников \\
\hline & Кадровая политика организации \\
\hline & Уровень внедрения технологий в организации по отношению к отрасли \\
\hline & Четкость целеполагания и планирования в организации \\
\hline \multirow{2}{*}{$\begin{array}{l}\text { 3. Внутри отрасли, но внеш- } \\
\text { ние к организации }\end{array}$} & Уровень конкуренции в отрасли \\
\hline & Наличие регулирующего органа в отрасли \\
\hline \multirow{2}{*}{$\begin{array}{l}\text { 4. Внутренние по отношению } \\
\text { к стране, но внешние по } \\
\text { отношению к отрасли }\end{array}$} & Макроэкономика страны \\
\hline & Наличие регулирующего органа в отрасли \\
\hline \multirow[t]{3}{*}{ 5. Международные факторы } & Миграция квалифицированной рабочей силы между странами \\
\hline & Эволюция передовых практик и технологических разработок \\
\hline & Макроэкономика мира \\
\hline
\end{tabular}

Международные

Национальные

Внутриотраслевые

Внутрифирменные

Внутриличностные

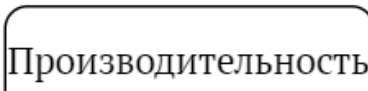

труда

Рисунок. Модель, показывающая влияние факторов на производительность труда 
Behrens, Kichko, and Thisse [7] считают, что в коротком периоде удаленная работа сначала привела к снижению производительности, затем она восстановилась и даже выросла, но в долгом периоде - предсказывают снижение производительности труда, так как работники не смогут обмениваться идеями и т. д.

Дополняя модель «зонтика факторов» по критерию возможности оказывать на них влияние, разделим факторы:

1) на внутренние, над которыми мы имеем контроль;

2) внешние, над которыми мы не имеем контроля. Однако возможна предварительная оценка событий, их визуализация и прогнозирование, что позволит принимать превентивные и корректирующие меры.

С учетом природы внутренних факторов, модель управления производительностью должна включать семь аспектов (табл. 3).

\section{Заключение}

Эффективное управление производительностью предполагает стандартизированную концепцию производительности, а также формирование единой и прозрачной методики ее измерения на отраслевом и организационном уровнях. Эта стандартизация станет сложным процессом и будет охватывать все необходимые параметры, влияющие на производительность предприятия. Внутри отрасли должны быть проведены опросы компаний, по которым будет возможно оценить детерминанты истинной производительности. Далее необходимо провести продольные и географические исследования, охватывающие данные о фирмах и отрасли. Если будут реализованы предложения, сформулированные выше, то будет обеспечено лучшее понимание феномена повышения производительности труда, что приведет к большему продвижению исследователями, менеджерами и политиками.

Проведенное исследование заключается в систематическом анализе материалов, доступных в научных журналах, и определении соответствующих факторов, влияющих на производительность труда. В ходе данной работы выполнена основная цель - объединены различные факторы для лучшего понимания сущности производительности и дальнейшего продвижения будущих исследований в области повышения производительности. В работе выделены 20 факторов производительности труда. Особое внимание уделено взаимодействию этих факторов в процессе управления производительностью.

Факторы производительности представлены в виде «зонтика», поскольку фактор более высокого уровня потенциально может влиять на факторы более низкого уровня. Например, изменения, происходящие в мировой экономике, неизбежно влияют (в той или иной степени) на производительность страны или отрасли, фирмы, или отдельного

Т а блица 3

Модель управления производительностью труда

\begin{tabular}{|c|c|}
\hline $\begin{array}{c}\text { Аспект модели управления } \\
\text { производительностью }\end{array}$ & Содержание \\
\hline 1. Фокус & $\begin{array}{l}\text { Понимание целей организации, возможности для тренировок. } \\
\text { Осведомлённость о последних технологических изменениях в мире } \\
\text { Политика приверженности, а не методы контроля }\end{array}$ \\
\hline 2. Стиль руководства & $\begin{array}{l}\text { Свобода принятия решений. } \\
\text { Лидерство высшего руководства } \\
\end{array}$ \\
\hline $\begin{array}{l}\text { 3. Организационная } \\
\text { структура }\end{array}$ & $\begin{array}{l}\text { Новаторская стимулирующая культура труда, сплоченный коллектив. } \\
\text { Достойный уровень оснащенности офиса, современное оборудование. } \\
\text { Различные социальные льготы, предоставляемые организацией (де-- } \\
\text { кретный отпуск, гибкий график, медицинское обслуживание, подарки) }\end{array}$ \\
\hline 4. Планирование & Общение и разделение ответственности \\
\hline 5. Адаптируемость & $\begin{array}{l}\text { Уровень цифровизации, автоматизации, ротация, расширение рабочих } \\
\text { мест }\end{array}$ \\
\hline $\begin{array}{l}\text { 6. Контроль и вознагражде- } \\
\text { ние }\end{array}$ & $\begin{array}{l}\text { Достойная оплата труда. } \\
\text { Система оценки, схема вознаграждений и признания. } \\
\text { Возможность карьерного роста }\end{array}$ \\
\hline $\begin{array}{l}\text { 7. Предпринимательская } \\
\text { культура }\end{array}$ & Схемы предложений, круг качества \\
\hline
\end{tabular}


человека. С другой стороны, факторы, связанные с работниками, отвечают за производительность на индивидуальном уровне и являются подмножеством производительности в масштабе всей организации. Аналогичным образом, факторы производительности в масштабах всей организации являются подмножеством производительности на уровне отрасли. Поэтому каждый фактор, находящийся на меньшем уровне, отвечает за производительность субъектов, присутствующих за его пределами.

\section{Библиографический список}

1. Симонова М. В. Управление производительностью труда в системе компетенций на региональном уровне // Вестник Воронежского государственного университета. Серия : Экономика и управление. 2020. № 4. С. 83-91.

2. Фомина Н. Н. О взаимосвязи дифференциации доходов населения и экономического развития региона // Вестник Воронежского государственного университета. Серия : Экономика и управление. 2019. № 4. C. $78-81$.

3. Abad J., Lafuente E., Vilajosana J. An assessment of the OHSAS 18001 certification process: objective drivers and consequences on safety performance and labour productivity // Safety Science. 2013. Vol. 60. P. 47-56.

4. Arthur J. B. Effects of human resource systems on manufacturing performance and turnover // Academy of Management Journal. 1994. Vol. 37 (3). P. 670-687.

5. Bala Subrahmanya M. H. Labour productivity, energy intensity and economic performance in small enterprises: A study of brick enterprises cluster in India // Energy conversion and management. 2006. Vol. 47 (6). P. $763-777$.

6. Beauregard T. A., Henry L. C. Making the link between work-life balance practices and organizational performance// Human Resource Management Review. 2009. Vol. 19 (1). P. 9-22.

7. Behrens K., Kichko S., Thisse J.-F. Working from Home: Too Much of a Good Thing? CESifo Working Paper No. 8831, 2021.

8. Bernolak I. Effective measurement and successful elements of company productivity: The basis of competitiveness and world prosperity // International Journal of Production Economics. 1997. Vol. 52 (1-2). P. 203-213.

9. Bower G. H., Hilgard E. R. Theories of Learning. Prentice Hall, NJ. 1981.

10. Bureš V., Stropková A. Labour productivity and possibilities of its extension by knowledge management aspects // Procedia - Social and Behavioral Sciences. 2014. Vol. 109. P. 1088-1093.
Выделенные факторы отражают семь аспектов управления производительностью, которые были положены в основу модели управления производительности труда. Результаты этого исследования могут помочь исследователям, менеджерам, заинтересованным в понимании концепции повышения производительности.

\section{Конфликт интересов}

Авторы декларируют отсутствие явных и потенциальных конфликтов интересов, связанных с публикацией настоящей статьи.

11. Calcagnini G., Giombini G., Travaglini G. Modelling energy intensity, pollution per capita and productivity in Italy: A structural VAR approach // Renewable and Sustainable Energy Reviews. 2016. Vol. 59. P. 1482-1492.

12. Conen W. S. 'Ageing and employers' perceptions of labour costs and productivity: a survey among European employers // International Journal of Manpower. 2012. Vol. 33 (6). P. 629-647.

13. Dall'Ora C., Ball J., Reinius M., Griffiths P. Burnout in nursing: a theoretical review // Human Resources for Health. 2020. Vol. 18.

14. Doloi H., Sawhney A., Iyer K. C., Rentala S. Analysing factors affecting delays in Indian construction projects // International Journal of Project Management. 2012. Vol. 30 (4). P. 479-489.

15. Durdyev S., Ismail S., Bakar N. A. Factors constraining labour productivity: case study of Turkmenistan // International Proceedings of Economics Development and Research. 2012. Vol. 55 (1). P. 1-5.

16. Enshassi A., Mohamed S., Mustafa Z. A., Mayer $P$. E. Factors affecting labour productivity in building projects in the Gaza Strip // Journal of Civil Engineering and Management. 2007. Vol. 13 (4). P. 245-254.

17. Feldstein $M$. Did wages reflect growth in productivity? // Journal of Policy Modelling. 2008. Vol. 30 (4). P. 591-594.

18. Gruman J. A., Saks A. M. Performance management and employee engagement // Human Resource Management Review. 2011. Vol. 21 (2). P. 123-136.

19. Gunasekaran A., Korukonda A. R., Virtanen I., YliOlli $P$. Improving productivity and quality in manufacturing organisations // International Journal of Production Economics. 1994. Vol. 36, no. 2. P. 169-183.

20. Hannula $M$. Total productivity measurement based on partial productivity ratios // International Journal of Production Economics. 2002. Vol. 78 (1). P. 57-67.

21. Hasan $R$. The impact of imported and domestic technologies on the productivity of firms: 
panel data evidence from Indian manufacturing firms // Journal of Development Economics. 2002. Vol. 69 (1). P. 23-49.

22. Huselid M. A. The impact of human resource management practices on turnover, productivity, and corporate financial performance // Academy of Management Journal. 1995. Vol. 38 (3). P. 635-672.

23. Koch M. J., McGrath R. G. Improving labor productivity: human resource management policies do matter // Strategic Management Journal. 1996. Vol. 17 (5). P. 335-354.

24. Künn-Nelen A., De Grip A., Fouarge D. Is parttime employment beneficial for firm productivity? // Industrial and Labor Relations Review. 2013. Vol. 66 (5). P. 1172-1191.

25. Kwong W. C., Toncich D., Lee S. F. Potential manufacturing productivity changes arising from the application of internet-based technologies // Journal of Materials Processing Technology. 2003. Vol. 139 (1). P. 35-39.

26. MacDuffie J. P., Kochan T. A. Does US firms invest less in human resources? Training in the world auto industry // Industrial Relations: A Journal of Economy and Society. 1995. Vol. 34 (2). P. 147-168.

27. Macey W. H., Schneider B. The meaning of employee engagement // Industrial and Organizational Psychology. 2008. Vol. 1 (1). P. 3-30.

28. Mathew J. The relationship of organizational culture with productivity and quality: a study of Indian software organizations // Employee Relations. 2017. Vol. 29 (6). P. 677-695.

29. Mefford R. N. Increasing productivity in global firms: the CEO challenge // Journal of International Management. 2019. Vol. 15 (3). P. 262-272.

30. Millea $M$., Fuess $S$. M. Does pay affect productivity or react to it? Examination of US manufacturing // Quarterly Review of Economics and Finance. 2005. Vol. 45 (4). P. 796-807.
31. Mohanty R. P. Consensus and conflicts in understanding productivity // International Journal of Production Economics. 1992. Vol. 28 (1). P. 95-106.

32. Nasirzadeh F., Nojedehi P. Dynamic modeling of labor productivity in construction projects // International Journal of Project Management. 2013. Vol. 31 (6). P. 903-911.

33. Nataraj $S$. The impact of trade liberalization on productivity: evidence from India's formal and informal manufacturing sectors // Journal of International Economics. 2011. Vol. 85 (2). P. 292-301.

34. Pandey M., Dong X. Y. Manufacturing productivity in China and India: the role of institutional changes // China Economic Review. 2009. Vol. 20 (4). P. 754-766.

35. Roberge $M$. É, van Dick $R$. Recognizing the benefits of diversity: when and how does diversity increase group performance? // Human Resource Management Review. 2010. Vol. 20 (4). P. 295-308.

36. Schonewille $M$. Does training generally work?: Explaining labour productivity effects from schooling and training // International Journal of Manpower. 2001. Vol. 22 (1/2). P. 158-172.

37. Wacker J. G., Yang C. L., Sheu C. Productivity of production labor, non-production labor, and capital: an international study // International Journal of Production Economics. 2006. Vol. 103 (2). P. 863-872.

38. Wagner J. The Causal Effects of Exports on Firm Size and Labor Productivity: First Evidence from a Matching Approach. HWWA Discussion Paper. 2001.

39. Wygant R. M. Improving productivity with financial incentives // Engineering Management International. 1987. Vol. 4 (2). P. 87-93.

40. Zhang J., Liu $X$. The evolving pattern of the wage-labor productivity nexus in China: evidence from manufacturing firm-level data // Economic Systems. 2013. Vol. 37 (3). P. 354-368.

41. Zwick T.Employee participation and productivity // Labour Economics. 2004. Vol. 11 (6). P. 715-740.
Гридасова Виктория Владимировна, магистрант кафедры экономической теории и мировой экономики, Воронежский государственный университет, Воронеж, Российская Федерация

E-mail:v.gridasova@bk.ru

ORCID ID: 0000-0002-7826-8993
Матершева Вера Викторовна, кандидат экономических наук, доцент кафедры экономической теории и мировой экономики, Воронежский государственный университет, Воронеж, Российская Федерация

E-mail: matersheva@mail.ru

ORCID ID: 0000-0001-8046-0725

Поступила в редакцию 14.01.2021

Подписана в печать 13.02.2021 


\title{
Pandemic and labor growth factors
}

\author{
V. V. Gridasova ${ }^{1}$, V. V. Matersheva ${ }^{2}$ \\ ${ }^{1,2}$ Voronezh State University, 1 University sq., 394018, Voronezh, Russian Federation
}

Cite as: Gridasova, V. V., Matersheva, V. V. (2021) Pandemic and labor growth factors. Proceedings of Voronezh State University. Series: Economics and Management. 1, 13-24. (In Russ., abstract in Eng.). DOI: 10.17308/ econ.2021.1/3346

\begin{abstract}
Importance. Labor productivity has become a very important concern for all economies focusing development through manufacturing. Russian scientists have already investigated this problem in sufficient detail. The present paper captures the idea of labor productivity from various research papers and identifies factors affecting labor productivity and their manifestation in a pandemic.

Objectives. This study consists of a systematic review of literature on labor productivity and is focused on finding a set of actionable factors which affect productivity. By the word 'actionable factors', we mean the factors upon which action can be taken to improve the productivity of the firm. There are two main objectives: 1 to study how the term productivity was defined by different authors 2 to find the set of those 'actionable factors' the presence or absence of which can improve the state of productivity for any organization.

Methods. In the process of achieving this objective, the methods of scientific knowledge were used: systematic review, analysis and synthesis. Systematic review helps in bridging the gap that traditional narrative reviews create due to use of personal, subjective and biased methodology by authors.

Conclusions and Relevance. Labor productivity in the manufacturing sector is a very complex process which is driven by numerous factors. Our systematic review shows this complexity as how one identified factor interacts or affects other identified factor. Managers must understand that for improving labor productivity, its true measurement is necessary. A true measurement shall reflect where the organization stands as compared to its competitors and must identify the gaps which it can close in order to improve its productivity. For the true measurement to happen, managers shall disseminate the importance of productivity measurement and improvement first to the top management and then to the whole employees.
\end{abstract}

Key words: factors labor productivity, classification, model of increasing labor productivity.

\section{Conflict of Interest}

The authors declare the absence of obvious and potential conflicts of interest related to the publication of this article.

\section{References}

1. Simonova, M. V. (2020) Labor productivity management in the competence system at the regional level. Proceedings of Voronezh State University. Series: Economics and Management. 4, 83-91. (In Russ., abstract in Eng.).

2. Fomina, N. N. (2019) [On interrelation of differentiation of population income and economic development of the region]. Proceedings of Voronezh State University. Series: Economics and Management. 4, 78-81. (In Russian).

3. Abad, J., Lafuente, E. \& Vilajosana, J. (2013) An assessment of the OHSAS 18001 certification process: objective drivers and consequences on safety performance and labour productivity. Safety Science. 60, 47-56.
4. Arthur, J. B. (1994) Effects of human resource systems on manufacturing performance and turnover. Academy of Management Journal. 37 (3), 670-687.

5. Bala Subrahmanya, M. H. (2006) Labour productivity, energy intensity and economic performance in small enterprises: A study of brick enterprises cluster in India. Energy conversion and management. 47 (6), 763-777.

6. Beauregard, T. A. \& Henry, L. C. (2009) Making the link between work-life balance practices and organizational performance. Human Resource Management Review. 19 (1), 9-22.

7. Behrens, K., Kichko, S. \& Thisse, J.-F. (2021) Working from Home: Too Much of a Good Thing? CESifo Working Paper No. 8831. 
8. Bernolak, I. (1997) Effective measurement and successful elements of company productivity: The basis of competitiveness and world prosperity. International Journal of Production Economics. 52 (1-2), 203-213.

9. Bower, G. H. \& Hilgard, E. R. (1981) Theories of Learning. Prentice Hall publ., NJ.

10. Bureš, V. \& Stropková, A. (2014) Labour productivity and possibilities of its extension by knowledge management aspects. Procedia - Social and Behavioral Sciences. 109, 1088-1093.

11. Calcagnini, G., Giombini, G. \& Travaglini, G. (2016) Modelling energy intensity, pollution per capita and productivity in Italy: A structural VAR approach. Renewable and Sustainable Energy Reviews. 59, 1482-1492.

12. Conen, W.S. (2012) "Ageing and employers" perceptions of labour costs and productivity: a survey among European employers. International Journal of Manpower. 33 (6), 629-647.

13. Dall'Ora, C., Ball, J., Reinius, M. \& Griffiths, P. (2020) Burnout in nursing: a theoretical review. Human Resources for Health. 18.

14. Doloi, H., Sawhney, A., Iyer, K.C. \& Rentala, S. (2012) “Analysing factors affecting delays in Indian construction projects”. International Journal of Project Management. 30 (4), 479-489.

15. Durdyev, S., Ismail, S. \& Bakar, N.A. (2012) Factors constraining labour productivity: case study of Turkmenistan. International Proceedings of Economics Development and Research. 55 (1), 1-5.

16. Enshassi, A., Mohamed, S., Mustafa, Z.A. \& Mayer, P.E. (2007) Factors affecting labour productivity in building projects in the Gaza Strip. Journal of Civil Engineering and Management. 13 (4), 245-254.

17. Feldstein, M. (2008) Did wages reflect growth in productivity?. Journal of Policy Modelling. 30 (4), 591-594.

18. Gruman, J.A. \& Saks, A.M. (2011) Performance management and employee engagement. Human Resource Management Review. 21 (2), 123-136.

19. Gunasekaran, A., Korukonda, A.R., Virtanen, I. \& Yli-Olli, P. (1994) Improving productivity and quality in manufacturing organizations. International Journal of Production Economics. 36 (2), 169-183.

20. Hannula, M. (2002) Total productivity measurement based on partial productivity ratios. International Journal of Production Economics. 78 (1), 57-67.

21. Hasan, R. (2002) “The impact of imported and domestic technologies on the productivity of firms: panel data evidence from Indian manufacturing firms". Journal of Development Economics. 69 (1), 23-49.

22. Huselid, M.A. (1995) The impact of human resource management practices on turnover, productivity, and corporate financial performance. Academy of Management Journal. 38 (3), 635-672.

23. Koch, M.J. \& McGrath, R.G. (1996) Improving labor productivity: human resource management policies do matter. Strategic Management Journal. 17 (5), 335-354.

24. Künn-Nelen, A., De Grip, A. \& Fouarge, D. (2013) Is part-time employment beneficial for firm productivity?. Industrial and Labor Relations Review. 66 (5), 1172-1191.

25. Kwong, W.C., Toncich, D. \& Lee, S.F. (2003) Potential manufacturing productivity changes arising from the application of internet-based technologies. Journal of Materials Processing Technology. 139 (1), 35-39.

26. MacDuffie, J.P. \& Kochan, T.A. (1995) Does US firms invest less in human resources? Training in the world auto industry. Industrial Relations: A Journal of Economy and Society. 34 (2), 147-168.

27. Macey, W.H. \& Schneider, B. (2008) The meaning of employee engagement. Industrial and Organizational Psychology. 1 (1), 3-30.

28. Mathew, J. (2017) The relationship of organisational culture with productivity and quality: a study of Indian software organizations. Employee Relations. 29 (6), 677-695.

29. Mefford, R.N. (2019) Increasing productivity in global firms: the CEO challenge. Journal of International Management. 15 (3), 262-272.

30. Millea, M. \& Fuess, S.M. (2005) Does pay affect productivity or react to it? Examination of US manufacturing. Quarterly Review of Economics and Finance. 45 (4) 796-807.

31. Mohanty, R.P. (1992) Consensus and conflicts in understanding productivity. International Journal of Production Economics. 28 (1), 95-106.

32. Nasirzadeh, F. \& Nojedehi, P. (2013) Dynamic modeling of labor productivity in construction projects. International Journal of Project Management. 31 (6), 903-911.

33. Nataraj, S. (2011) The impact of trade liberalization on productivity: evidence from India's formal and informal manufacturing sectors. Journal of International Economics. 85 (2), 292-301.

34. Pandey, M. \& Dong, X.Y. (2009) Manufacturing productivity in China and India: the role of institutional changes. China Economic Review. 20 (4), 754766.

35. Roberge, M.É., \& van Dick, R. (2010) Recognizing the benefits of diversity: when and how does diversity increase group performance?. Human Resource Management Review. 20 (4), 295-308.

36. Schonewille, M. (2001) Does training generally work?: Explaining labour productivity effects from schooling and training. International Journal of Manpower. 22 (1/2), 158-172.

37. Wacker, J.G., Yang, C.L. \& Sheu, C. (2006) Productivity of production labor, non-production labor, and capital: an international study. International Journal of Production Economics. 103 (2), 863872 . 
38. Wagner, J. (2001) The Causal Effects of Exports on Firm Size and Labor Productivity: First Evidence from a Matching Approach. HWWA Discussion Paper.

39. Wygant, R.M. (1987) Improving productivity with financial incentives. Engineering Management International. 4 (2), 87-93.
40. Zhang, J. \& Liu, X. (2013) The evolving pattern of the wage-labor productivity nexus in China: evidence from manufacturing firm-level data. Economic Systems. 37 (3), 354-368.

41.Zwick, T. (2004) Employee participation and productivity. Labour Economics. 11 (6), 715-740.
Victoria V. Gridasova, Master Student of the Department of Economic Theory and World Economy, Voronezh State University, Voronezh, Russian Federation

E-mail: v.gridasova@bk.ru

ORCID ID: 0000-0002-7826-8993
Vera V. Matersheva, Cand. Sci. (Econ.), Assoc. Prof. Department of Economic Theory and World Economy, Voronezh State University, Voronezh, Russian Federation

E-mail:nmya@gmail.com

ORCID ID: 0000-0001-8046-0725

Received 14.01.2021

Accepted 13.02.2021 